\title{
Duality of the Incompatibility Tensor
}

\author{
Kazuhito Yamasaki ${ }^{1, *}$ and Tadashi Hasebe ${ }^{2}$ \\ ${ }^{1}$ Department of Planetology, Kobe University, Kobe 657-8501, Japan \\ ${ }^{2}$ Department of Mechanical Engineering, Kobe University, Kobe 657-8501, Japan
}

Disclination resulting from kink deformation of the long-period stacking-ordered phase of $\mathrm{Mg}-\mathrm{Zn}-\mathrm{Y}$ alloy affects the strength of the alloy. In this study, we examined theoretical aspects of the kinematic equation of defects, including dislocations and disclinations, based on the dual structure of strain and stress space. Two types of disclination, related to dislocation and bend-twist, were identified. Disclination types were distinguished based on incompatibility. In "dual strain space", we show that incompatibility is due to a generalized stress function, i.e., the Beltrami stress function, which is a three-dimensional version of the Airy stress function that includes non-diagonal components. [doi:10.2320/matertrans.MT-MM2019004]

(Received September 2, 2019; Accepted December 17, 2019; Published January 31, 2020)

Keywords: incompatibility, duality, disclination, stress function, kink deformation

\section{Introduction}

Recently, there has been much interest in the $\mathrm{Mg}-\mathrm{Zn}-\mathrm{Y}$ alloy developed by Kawamura, ${ }^{1)}$ due to its excellent properties including high specific strength. ${ }^{2}$ This strength property originates from the long-period stacking-ordered (LPSO) phase of the $\mathrm{Mg}-\mathrm{Zn}-\mathrm{Y}$ lattice, ${ }^{3-5)}$ characterized by a particular kink structure. ${ }^{6-8)}$ Kink deformation of the LPSO phase is essential for the excellent mechanical properties demonstrated by the $\mathrm{Mg}-\mathrm{Zn}-\mathrm{Y}$ alloy. ${ }^{9}$ )

Normal kink deformation is part of the dislocation line of the slip plane. However, it has been pointed out that not only dislocations, but also disclinations, affect the strength of the LPSO phase. For instance, Inamura ${ }^{9}$ showed theoretically that disclination plays an important role in the connection of kink bands, according to the geometric compatibility (rank-1 tensor). The incompatibility tensor has attracted interest for crystal plasticity-based finite element simulations, where kink deformation has been seen in $\mathrm{Mg}$ alloys with a millefeuille-structured LPSO phase. ${ }^{10,11)}$ The relationship between incompatibility and disclination is expected to be key to understanding kink deformation in the LPSO phase. However, there have been few theoretical studies of this phenomenon.

The purpose of this paper is to review the theoretical aspects of the incompatibility tensor using the kinematic equation of defects, accounting for both dislocations and disclinations. To achieve this, we examined the dual structure of strain and stress space based on differential geometry. ${ }^{12,13)}$ This differential geometrical approach is useful for deriving kinematic and continuity equations of defects in a systematic way, and has been applied to various complicated deformations with defect fields, as reported in Earth science. ${ }^{14,15)}$

The structure of this paper is as follows. In Section 2, we consider incompatibility in strain space and derive the relationship between incompatibility and disclinations to reveal its dual structure, i.e., the stress space. In Section 3, we reconsider the incompatibility in "dual stress space"; our analysis shows that the concept of incompatibility can be

*Corresponding author, E-mail: yk2000@kobe-u.ac.jp extended to generalized stress functions. Conclusions are presented in Section 4.

\section{Incompatibility in Strain Space}

First, we review the incompatibility in strain space, based on a differential geometrical approach, ${ }^{13)}$ and consider how it is related to disclination. From a geometrical perspective, the strain space structure is characterized by metric, because this is the basic quantity from which other important quantities can be derived, such as curvature. In strain space, the metric and the curvature correspond to distortion and disclination, respectively, ${ }^{12,13)}$ and the geometrical order of the incompatibility corresponds to the curvature. ${ }^{10)}$ From this we can derive the incompatibility using the basic quantity of strain space, i.e., distortion.

In the differential form, distortion $\beta$ and incompatibility $\eta$ correspond to 1-form and 3-form, respectively. Given that the exterior derivative operator $d$ maps $p$-form to $(p+1)$-form, the operator $d$ is applied to $\beta$ twice to derive $\eta$. Because $d d(\cdots)=0$, we cannot obtain the incompatibility simply using the expression $d d \beta$. As such, we assume the following:

$$
\eta=d \beta \overleftarrow{d}
$$

where $\overleftarrow{d}$ is the operator acting from the right simultaneously. Since $d$ (1-form) corresponds to $\nabla \times(\cdots)$ in the nabla notation, eq. (1) corresponds to $\eta=\nabla \times \beta \times \nabla$. The index notation of this nabla expression is given by

$$
\eta_{i j}=\epsilon_{j m n} \epsilon_{i k l} \partial_{m} \partial_{k} \beta_{n l},
$$

where $\epsilon_{i j k}$ is the permutation tensor and $\partial_{i}=\partial / \partial x^{i}$ in which the Einstein summation convention is used. This is in agreement with the previous index notation of incompatibility. ${ }^{10)}$ Here, $\eta$ in eq. (1) is referred to as the incompatibility in strain space.

The next expression also uses the operator $d$ twice: $d^{*} d \beta$, where ${ }^{*}$ is the Hodge star operator in three-dimensional space. In three-dimensional space, the operator ${ }^{*}$ maps $p$ form to (3-p)-form; thus, the form number of $* d \beta$ is [3$(1+1)=1]$. The above expression corresponds to $\nabla \times$ $(\nabla \times \beta)$ in nabla notation. The index notation of this nabla 
expression is given by $\epsilon_{i m n} \epsilon_{n k l} \partial_{m} \partial_{k} \beta_{l j}$. Note that the index $n$ appears twice in the permutation tensor and it is equal to $\partial_{i} \partial_{l} \beta_{l j}-\partial_{k} \partial_{k} \beta_{i j}$ based on the well-known identity $\epsilon_{i m n} \epsilon_{n k l}=$ $\delta_{i k} \delta_{m l}-\delta_{i l} \delta_{m k}$, where $\delta_{i j}$ is Kronecker delta. Therefore, the index notation of $\nabla \times(\nabla \times \beta)$ is not equal to the incompatibility (2). However, some papers use this nabla notation for the index notation (2). To avoid any such confusion, here we use only the index notation of the incompatibility (2).

Next, we consider eq. (1) based on the kinematic equation of defects. According to the differential geometrical approach to defects, the dislocation density 2 -form $\alpha$ is given by $\alpha=d \beta+\kappa$, where $\beta$ is the distortion 1 -form defined above, and $\kappa$ is the bend-twist 2 -form. ${ }^{12,13)}$ Substitution of this kinematic equation into eq. (1) gives

$$
\eta=\theta^{\alpha}-\theta^{\kappa}
$$

where $\theta^{\alpha}$ and $\theta^{\kappa}$ represent the disclination density 3-form obtained from the dislocation density (i.e., $\alpha \overleftarrow{d}=\theta^{\alpha}$ ) and bend-twist (i.e., $\kappa \overleftarrow{d}=\theta^{\kappa}$ ), respectively. Notably, in our previous studies, ${ }^{12,13)} \theta^{\alpha}$ was not distinguished from $\theta^{\kappa}$. However, eq. (3) shows that when incompatibility exists, these two entities are not equal to each other. In other words, the incompatibility in strain space leads to a quantitative difference between $\theta^{\alpha}$ and $\theta^{\kappa}$. Of course, disclination can be recognized as one of the incompatibility forms, if we ignore bend-twist in the mathematical analysis of the defect field (i.e., $\theta^{\kappa}=0$ ).

Kadic and Edelen ${ }^{16)}$ divides the dislocations into two types, i.e., translational and disclination-driven dislocations, by noting the homogeneity breaking of translation group $\mathrm{T}(3)$ and rotational group $\mathrm{SO}(3)$ movements in material space. In this paper, the former and the latter dislocation types are related to $H \theta^{\alpha}$ and $H \theta^{\kappa}$, respectively, where $\mathrm{H}$ is the homotopy operator, specifically, the inverse of the exterior derivative operator. ${ }^{17)}$ Since $\mathrm{H}$ is a linear operator, ${ }^{17)}$ eq. (3) means that $H \eta$ quantifies the difference in contribution between $\mathrm{T}(3)$ and $\mathrm{SO}(3)$. Hasebe et al. ${ }^{10)}$ has pointed out that the incompatibility is due to pure deformation and pure rotation. The results imply that the incompatibility provides information about the group related to the defect field, such as $\mathrm{T}(3) \triangleright \mathrm{SO}(3)$, a six-parameter gauge group that leaves the Lagrangian invariant of deformation field with defects. ${ }^{18)}$

\section{Incompatibility in Stress Space}

In Section 2, we consider the relationship between distortion and incompatibility in strain space. According to previous studies, ${ }^{12,13)}$ strain space has a dual structure, i.e., the stress space. The metric of the stress space is a stress function, similar to the distortion found in strain space. Analogous to eq. (1), we assumed dual incompatibility 3form in dual stress space, defined by

$$
\eta^{\sigma}=d \psi \overleftarrow{d}
$$

where $\psi$ is the stress function 1-form in dual stress space. Using a similar form to that given in Section 2, the index notation corresponds to that of (4), given by

$$
\eta_{i j}^{\sigma}=\epsilon_{j m n} \epsilon_{i k l} \partial_{m} \partial_{k} \psi_{n l} .
$$

To clarify the physical meaning of (5), we examine a particular example of this expression. When we consider the diagonal component of $\psi$, i.e., $n=l$, eq. (5) becomes $\eta_{i j}^{\sigma}=$ $\partial_{k} \partial_{k} \delta_{i j} \psi-\partial_{i} \partial_{j} \psi$ where we set $\psi_{11}=\psi_{22}=\psi_{33}=\psi$ for simplicity. Then, we obtain

$$
\eta_{12}^{\sigma}=-\partial_{1} \partial_{2} \psi, \quad \eta_{11}^{\sigma}=\partial_{2} \partial_{2} \psi+\partial_{3} \partial_{3} \psi .
$$

In two-dimensional space, (6) becomes

$$
\eta_{12}^{\sigma}=-\partial_{1} \partial_{2} \psi, \quad \eta_{11}^{\sigma}=\partial_{2} \partial_{2} \psi .
$$

This is in agreement with the well-known Airy stress function, in which (6) is a particular component of the threedimensional version of the Airy stress function, i.e., the Maxwell stress function. Thus, the dual incompatibility in stress space can be recognized as stress. In this case, (5) can be interpreted as the generalized stress function, i.e., the Beltrami stress function. For example, the component of (5) is given by

$$
\begin{aligned}
& \eta_{12}^{\sigma}=-\partial_{3} \partial_{3} \psi_{12}-\partial_{1} \partial_{2} \psi+\partial_{1} \partial_{3} \psi_{23}+\partial_{2} \partial_{3} \psi_{31}, \\
& \eta_{11}^{\sigma}=\partial_{2} \partial_{2} \psi+\partial_{3} \partial_{3} \psi-2 \partial_{2} \partial_{3} \psi_{23} .
\end{aligned}
$$

Comparing (8) with (6) and (7), we can confirm that the Beltrami stress function is the three-dimensional version of the Airy stress function with non-diagonal components, $\psi_{12}$, $\psi_{23}$ and $\psi_{31}$.

In Section 2, we showed that the incompatibility in strain space is closely related to the disclination field. In this section, we consider the duality of the incompatibility and derive the Beltrami stress function. The results imply that we should consider the Beltrami stress function in the defect field, including disclination.

Studies on two-dimensional elasticity have shown that the Airy stress function is useful for analyzing deformation, including the dislocation field. ${ }^{19,20)}$ Hasebe et al., ${ }^{10)}$ uses the Maxwell stress function (a three-dimensional version of the Airy stress function) to model and simulate the dislocation substructure. In future studies, we will consider the Beltrami stress function that generalizes the Maxwell stress function, to include non-diagonal components for analyzing the highstrength kink deformation in the LPSO phase.

\section{Conclusions}

Our main conclusions are as follows.

(1) We considered the incompatibility in strain space whose metric is the distortion. The expression obtained indicates that when incompatibility exists, there are two types of disclinations: $\theta^{\alpha}$ and $\theta^{\kappa}$, where $\theta^{\alpha}$ is caused by dislocation, and $\theta^{\kappa}$ is caused by bend-twist. When incompatibility vanishes, we obtain $\theta^{\alpha}=\theta^{\kappa}$, as assumed in previous papers.

(2) We also consider the incompatibility in "dual strain space", i.e., the stress space whose metric is the stress function. This dual incompatibility corresponds to the generalized stress function (Beltrami stress function) that is the three-dimensional version of the Airy stress function including non-diagonal components. 


\section{Acknowledgments}

This work was supported by JSPS KAKENHI Grant Number $19 \mathrm{H} 05129$.

\section{REFERENCES}

1) Y. Kawamura, K. Hayashi, A. Inoue and T. Masumoto: Mater. Trans. 42 (2001) 1172-1176.

2) H. Okuda, M. Yamasaki, Y. Kawamura, M. Tabuchi and H. Kimizuka: Sci. Rep. 5 (2015) 14186.

3) E. Abe, Y. Kawamura, K. Hayashi and A. Inoue: Acta Mater. 50 (2002) 3845-3857.

4) M. Matsuda, S. Ando and M. Nishida: Mater. Trans. 46 (2005) 361-364.

5) D. Egusa and E. Abe: Acta Mater. 60 (2012) 166-178.

6) K. Hagihara, T. Okamoto, H. Izuno, M. Yamasaki, M. Matsushita, T. Nakano and Y. Kawamura: Acta Mater. 109 (2016) 90-102.

7) J.K. Kim, S. Sandlobes and D. Raabe: Acta Mater. 82 (2015) 414-423.

8) K. Hagihara, M. Honnami, R. Matsumoto, Y. Fukusumi, H. Izuno, M.
Yamasaki, T. Okamoto and Y. Kawamura: Mater. Trans. 56 (2015) 943-951.

9) T. Inamura: Acta Mater. 173 (2019) 270-280.

10) T. Hasebe, M. Sugiyama, H. Adachi, S. Fukutani and M. Iida: Mater. Trans. 55 (2014) 779-787.

11) T. Kitano, Y. Nawa and T. Hasebe: The Computational Mechanics Conference, (2018) p. 298.

12) K. Yamasaki and H. Nagahama: J. Phys. A 32 (1999) L475.

13) K. Yamasaki and H. Nagahama: J. Phys. A 35 (2002) 3767.

14) R. Teisseyre: Bull. Seismol. Soc. Am. 101 (2011) 1683-1691.

15) R. Abreu, C. Thomas and S. Durand: Phys. Earth Planet. Inter. 276 (2018) 215-225.

16) A. Kadic and D.G. Edelen: A Gauge Theory of Dislocations and Disclinations, (Springer, Berlin, 1983).

17) D.G. Edelen and D.C. Lagoudas: Gauge Theory and Defects in Solids, (Elsevier, Amsterdam, 2012)

18) A. Kadic and D.G. Edelen: Int. J. Eng. Sci. 20 (1982) 433-438.

19) K. Yamasaki and T. Yajima: Acta Geophys. 60 (2012) 4-23.

20) A.I. Kalandiya: Mathematical Methods of Two-dimensional Elasticity, (Mir Publishers, Moscow, 1975). 